\title{
Crónica
}

\section{ACTA DE LA COMISIÓN DE PREMIOS PARA EL AÑO 2004}

En Santiago de Chile, con fecha 16 de Agosto de 2005, a las 12:30 horas, se reúne la Comisión de Premios formada de acuerdo a los artículos 26, 27 y 29 de los Estatutos de la Sociedad Chilena de Obstetricia y Ginecología.

El Jurado constituido por el Presidente de la Sociedad Dr. Enrique Oyarzún E. por los miembros de la Comisión Dr. Mario Herrera M. y Dr. Carlos Ramírez R. y por el Secretario General Dr. Eugenio Suárez P., éste último con derecho a voz solamente acuerdan otorgar los siguientes premios correspondientes al año 2004:

Premio "DR. JORGE DÍAZ BRAVO" año 2004 al mejor trabajo de Ingreso a la Sociedad Chilena de Obstetricia y Ginecología: Se consideraron los 5 trabajos de ingreso presentados en ese año. El premio fue para "IVS POSTERIOR (POSTERIOR INTRAVAGINAL SLINGPLASTY) PARA EL TRATAMIENTO DEL PROLAPSO DE CUPULA VAGINAL O PROCIDENCIA DE UTERO: SERIE DE CASOS" de los Drs. Alfredo Silva R., Luis Ferrada C., Carlos Arroyo M., y Kinovape Nalbandian L. de la Unidad de Uroginecología, Servicio de Obstetricia y Ginecología, Hospital San Juan de Dios y Servicio de Obstetricia y Ginecología, Hospital de Castro.

Premio "DR. JUAN MALFANTI" año 2004 al mejor trabajo de casuística, al trabajo: "ALTERNATIVA QUIRUGICA EN LA HEMORRAGIA GRAVE DEL PUERPERIO" de los Drs. Gabriel Mitelman M., Gino La Rosa A., Fernando Martínez A., Roberto Cadima R. Servicio de Ginecología y Obstetricia, Hospital Félix Bulnes C.

Premio "DR. JUAN WOOD WALTERS" año 2004, al mejor trabajo de Ginecología al trabajo: "DISTRIBUCIÓN DE LA PATOLOGIA ANEXIAL EN MUJER CHILENA. EXPERIENCIA DE LA UNI-
VERSIDAD CATOLICA DE CHILE" de Ios Drs. Mauricio Cuello F., Paulina Merino O., Alejandra Etchegaray B., Juan Pablo Ortega R., Cristián Pomés C., Nicanor Barrena G., Roger Gejman E. Departamento de Obstetricia y Ginecología, Pontificia Universidad Católica de Chile. Servicio de Obstetricia y Ginecología, Hospital San Borja Arriarán, Universidad de Chile. Departamento de Anatomía Patológica, Pontificia Universidad Católica de Chile

MENCIÓN HONROSA al premio Dr. Juan Wood Walters 2004 para el trabajo: "ESTUDIO DE VARIABLES CLINICAS Y METABOLICAS EN MUJERES CON HIPERANDROGENISMO CLINICO" de los Drs. Sergio Cheviakoff Z., Sergio Carmona G., y Rodolfo Lahsen M. de la P., Departamento de Obstetricia y Ginecología, Unidad de Endocrinología, Hospital Clínico J.J. Aguirre Facultad de Medicina, Universidad de Chile.

Premio "Dr. VÍCTOR MANUEL GAZITÚA GUZMÁN" año 2004 al mejor trabajo de Obstetricia al trabajo: "AUSENCIA DE TRANSMISION PERINATAL DE VIH EN 40 EMBARAZADAS TRATADAS CON TERAPIA ANTI-RETROVIRAL DE ALTA POTENCIA" de los Drs. Fernando Abarzúa C., Carlos Pérez C. Claudio Callejas C, Jean Cyr Zombi y Bernard Vandercam, Departamento de Obstetricia y Ginecología, Departamento de Medicina Interna, Pontificia Universidad Católica de Chile, Departamento de Medicina Interna e Infectología y Enfermedades Tropicales, Cliniques Universitaires Saint Luc, Université Catolique de Lovaine.

MENCIÓN HONROSA al premio Dr. Víctor Manuel Gazitúa Guzmán 2004 para el trabajo: DESIGUALDAD EN MORTALIDAD PERINATAL 
ENTRE LAS COMUNAS DE LA PROVINCIA DE SANTIAGO" del Dr. Enrique Donoso S. Departamento de Obstetricia y Ginecología, Pontificia Universidad Católica de Chile.

Premio "DR. CARLOS MONCKEBERG BRAVO" año 2004 al mejor trabajo de Perinatología: Este premio se declara desierto.

La Comisión deja constancia que hay muchos trabajos merecedores de premios, pero los auto- res no son miembros de la Sociedad Chilena de Obstetricia y Ginecología.

Para dejar constancia firman:

Drs. CARLOS RAMÍREZ R., MARIO HERRERA M.

Drs. EUGENIO SUAREZ P., ENRIQUE OYARZUN E. Secretario General Presidente 